\title{
Tecnologia da informação e comunicação na gestão de grupos de pesquisa em enfermagema
}

\author{
Information and communication technology in the management of Nursing research groups \\ Tecnología de información y comunicación em la gestión de grupos de investigación em enfermería
}

\section{Greici Capellari Fabrizzio ${ }^{1}$ (]) \\ Juliana Martins Ferreira ${ }^{1}$ (1) \\ Daniele Cristina Perin ${ }^{1}$ (D) \\ Patricia Klock ${ }^{1}$ (1)}

Alacoque Lorenzini Erdmann ${ }^{1}$ (1)

José Luís Guedes dos Santos ${ }^{1}$ (B)

1. Universidade Federal de Santa Catarina, Departamento de Enfermagem. Florianópolis, SC, Brasil.
Autor correspondente:

Greici Capellari Fabrizzio.

E-mail: greicicapellari@hotmail.com.

Recebido em 12/08/2020.

Aprovado em 06/11/2020.

DOl:https://doi.org/10.1590/2177-9465-EAN-2020-0299

\section{Resumo}

Objetivo: este estudo teve como objetivo compreender como as Tecnologias da Informação e Comunicação são utilizadas na gestão de grupos de pesquisa de enfermagem. Método: trata-se de uma pesquisa qualitativa ancorada teórica e metodologicamente na Teoria Fundamentada nos Dados (perspectiva straussiana), tendo como cenário de estudo 14 grupos de pesquisa vinculados a um Programa de Pós-Graduação em Enfermagem. A amostragem teórica do estudo foi composta por 21 pesquisadores. Os dados foram coletados por meio de entrevistas e analisados a partir do sistema de codificação. Resultados: as Tecnologias de Informação e Comunicação são utilizadas pelos grupos de pesquisa para facilitar os processos de gestão e o desenvolvimento das pesquisas. Dividem-se em três funções principais: comunicação e armazenamento (Whatsapp ${ }^{\circledR}$ e e-mail), estratégias de visibilidade (site e mídias sociais) e estratégia operacional (software de análise de dados). Conclusão e implicações para a prática: as Tecnologias de Informação e Comunicação constroem um elo entre a tecnologia e a construção do conhecimento científico, tecnológico e de inovação. Os Grupos de Pesquisa podem intensificar ainda mais o uso dessas ferramentas, tanto para recrutamento de amostra como para o desenvolvimento de redes colaborativas de pesquisa.

Palavras-chave: Gestão da informação; Grupos de pesquisa; Tecnologia da informação e comunicação; Gestão de ciência, tecnologia e inovação em saúde; Pesquisa em Enfermagem.

\section{Abstract}

Objective: This study aimed to understand how Information and Communication Technologies are used in the management of Nursing research groups. Method: This is a qualitative research based on theory and methodology in the Grounded Theory (direct perspective), with the study setting being 14 research groups linked to a Graduate Program in Nursing. The theoretica sample of the study was composed of 21 researchers. Data was collected through interviews and analyzed using the coding system. Results: Information and Communication Technologies are used by research groups to facilitate research management and development processes. They are divided into three main functions: communication and storage (Whatsapp ${ }^{\circledR}$ and e-mail), visibility strategies (website and social media) and operational strategy (data analysis software). Conclusion and implications for the practice: Information and Communication Technologies build a link between technology and the construction of scientific, technological and innovation knowledge. Research groups can further intensify the use of these tools, both for recruiting samples and for developing collaborative research networks.

Keywords: Information management; Research groups; Information Technology; Health Sciences, technology, and innovation management; Nursing Research

\section{RESUMEN}

Objetivo: este estudio tuvo como objetivo comprender cómo se utilizan las Tecnologías de la Información y la Comunicación en la gestión de grupos de investigación en enfermería. Método: investigación cualitativa con base teórica y metodológica en la Teoría Fundamentada en los Datos (perspectiva straussiana), con un estudio realizado con 14 grupos de investigación vinculados a un Programa de Posgrado en Enfermería. La muestra teórica del estudio estuvo compuesta por 21 investigadores. Los datos fueron recolectados a través de entrevistas y analizados utilizando el sistema de codificación. Resultados: los grupos de investigación utilizan las Tecnologías de la Información y la Comunicación para facilitar la gestión de la investigación y los procesos de desarrollo. Dichas tecnologías cumplen tres funciones principales: comunicación y almacenamiento (Whatsapp ${ }^{\circledR}$ y correo electrónico), estrategias de visibilidad (sitio web y redes sociales) y estrategia operativa (software de análisis de datos). Conclusión e implicaciones para la práctica: las Tecnologías de la Información y la Comunicación crean un vínculo entre una tecnología y una construcción de conocimiento científico, tecnológico y de innovación. Los grupos de investigación pueden intensificar aún más el uso de estas herramientas, tanto para reclutar muestras como para desarrollar redes de investigación colaborativas.

Palabras clave: Gestión de información; Grupos de investigación; Tecnología de la información; Gestión de ciencia, Tecnología e innovación en salud; Investigación en Enfermería.

${ }^{a}$ Artigo extraído da dissertação de mestrado Gestão de grupos de pesquisa de um programa de pós-graduação em enfermagem stricto sensu de uma universidade pública, de autoria de Greici Capellari Fabrizzio, orientada por Alacoque Lorenzini Erdmann, Programa de Pós-graduação em Enfermagem da Universidade Federal de Santa Catarina, defesa no ano de 2018. 


\section{INTRODUÇÃO}

Com o passar dos anos, a educação vem passando por modificações que a transformaram de um sistema tradicional de ensino para um ambiente dinâmico e criativo, em que os estudantes são estimulados para o desenvolvimento de descobertas, ao mesmo tempo em que aprendem. ${ }^{1}$ Muitas dessas transformações são incitadas pelo uso de tecnologias, dentre elas, a Tecnologia da Informação e Comunicação.

Desde o surgimento das tecnologias, ocorreram rápidas transformações em todos os âmbitos da sociedade, resultado em alterações em diversos cenários, como, por exemplo: na educação, na economia, no meio ambiente, na saúde, entre outros. As universidades e centros de pesquisas, mais especificamente os grupos de pesquisa, não se mantiveram inerte a esse cenário de mudanças e passaram a incorporar as suas práticas algumas dessas tecnologias disponíveis.

No Brasil, os Grupos de Pesquisa em Enfermagem foram criados em 1970 e, desde então, principalmente a partir do ano de 2002, houve um crescimento exponencial no que se refere a sua expansão. No ano 2000, eram 1.832 grupos na grande área de Ciências da Saúde. Em 2016, esse número passou para 5.877 grupos de pesquisa. $^{2}$

Com a expansão dos grupos de pesquisa, fez-se necessário o estabelecimento de processos de gestão para o suprimento de demandas advindas dos pesquisadores, das exigências da formação, das necessidades de pesquisa, bem como das agências de fomento e da exigência por grupos extremamente produtivos.

Para tanto, os processos de gestão em grupos de pesquisa contam com o apoio da Tecnologia da Informação e Comunicação. Um conceito de Tecnologia da Informação e Comunicação ampliado, adotado para este trabalho e intimamente relacionado com os princípios da gestão, engloba a utilização de tecnologias para auxiliar no desenvolvimento de atividades humanas, sejam elas para o público em geral ou institucional, ligadas à informação, ao processamento, ao armazenamento e a disseminação de informações. ${ }^{3}$ As Tecnologias da Informação e Comunicação auxiliam para que os processos de gestão se tornem mais ágeis, interativos e flexíveis, a fim de acompanhar as demandas exigidas pela ciência. ${ }^{4}$

A integração de Tecnologias da Informação e Comunicação está intimamente relacionada à inovação e excelência em grupos de pesquisa. Essas tecnologias estão presentes na produção do conhecimento científico, mas também nas questões relacionadas à informação, como a produção, disseminação e o seu consumo. A maturação das Tecnologias de Informação e Comunicação coloca-se como um ponto indispensável para que um grupo de pesquisa atinja o seu ponto mais alto na produção do conhecimento. ${ }^{4}$

Nos grupos de pesquisa, a difusão e uso de Tecnologias da Informação e Comunicação tornaram-se indispensáveis pelas demandas advindas da produção do desenvolvimento conhecimento científico e tecnológico, por uma influência que advém do mercado e de uma maior difusão no meio da economia. Além disso, estão relacionadas a processos mais intensos e rápidos com tecnologias mais complexas e a expansão de tecnologias disponíveis para a inovação.

As Tecnologias da Informação e Comunicação contribuem para o estabelecimento de parcerias entre grupos de pesquisa, empresas, indústrias, escolas, comunidades e entre pesquisadores, possibilitando o compartilhamento sistematizado de informações, que são de interesse comum, facilitando o entendimento e a solução de problemas para determinada área de conhecimento. As aplicações das Tecnologias de Informação e Comunicação nos grupos de pesquisa trazem como aspectos positivos: a eficiência da comunicação; a interação com os sistemas externos; a facilidade na exploração de oportunidades de pesquisa sobre diversos temas. ${ }^{4}$

A literatura prévia sobre o conteúdo, sugere pesquisas que abordem a temática da tecnologia em diversos temas e especialidades da área da saúde, comparando a utilização de mais de um recurso tecnológico e avaliando os desfechos primários e secundários das tecnologias empregadas na educação a longo prazo. ${ }^{5}$ No entanto, não foram identificados estudos relacionados especificamente à adoção de Tecnologias da Informação e Comunicação na gestão de grupos de pesquisa de enfermagem.

Diante do exposto, o presente estudo teve como objetivo compreender como as Tecnologias da Informação e Comunicação são utilizadas na gestão de grupos de pesquisa de enfermagem.

\section{MÉTODO}

Pesquisa qualitativa, com aporte teórico-metodológico da Teoria Fundamentada nos Dados (TFD), vertente straussiana. ${ }^{6}$ Os resultados proeminentes deste estudo estão inseridos no projeto intitulado: Gestão de Grupos de pesquisa de um Programa de Pós-Graduação em Enfermagem stricto sensu de uma universidade pública".

O cenário do estudo foram 14 grupos de pesquisa vinculados a um Programa de Pós-Graduação em Enfermagem stricto sensu de uma universidade pública do sul do país.

A amostragem teórica do estudo foi composta por 21 pesquisadores vinculados aos grupos de pesquisa e conhecedores do seu funcionamento e da utilização das Tecnologias da Informação e Comunicação. Os participantes foram dispostos em dois grupos amostrais: o primeiro grupo amostral foi composto por dez líderes de grupos de pesquisa; o segundo grupo amostral foi formado por nove discentes e por dois ex-líderes de grupos de pesquisa. A partir das inferências analíticas baseadas nos dados do primeiro grupo amostral, emergiu a hipótese de uma gestão compartilhada entre líderes e participantes de grupos de pesquisa, aprofundada no segundo grupo amostral. O uso das Tecnologias da Informação e Comunicação para a gestão de grupos de pesquisa em enfermagem perpassou os dois grupos amostrais.

A amostra foi composta por participantes que atenderam aos critérios de inclusão propostos. Para o primeiro grupo amostral: atuação como docente permanente, líder ou vice-líderes de grupo de pesquisa por no mínimo dois anos, orientadores de mestrado e doutorado. Tendo como critérios de inclusão para o segundo grupo amostral: discentes, participantes de grupo 
de pesquisa por no mínimo seis meses, docentes voluntários e ex-líderes de grupos de pesquisa. Os critérios de exclusão para ambos os grupos amostrais foram: estar em férias, afastado do trabalho ou da Universidade por qualquer motivo ou em atividades acadêmicas fora do país durante a coleta de dados.

Quanto à composição da amostra do primeiro grupo amostral, dos 29 líderes de grupos de pesquisa, dez deles foram excluídos por não preencherem aos critérios de inclusão do estudo, oito não responderam ao convite para participação na pesquisa e um se recusou a participar do estudo. O segundo grupo amostral foi formado por nove discentes e por dois ex-líderes de grupos de pesquisa, os 11 participantes aceitaram participar do estudo e preencheram os critérios de inclusão. Em nenhum dos grupos amostrais houve desistências.

A coleta de dados ocorreu de abril a outubro de 2018. O convite para participação na pesquisa foi realizado via e-mail obtido no site do programa de pós-graduação e foram informados sobre os objetivos por meio do Termo de Consentimento Livre e Esclarecido. Após o aceite, foi verificado uma data, horário e local adequados aos participantes e ao pesquisador para realização da entrevista. A fim de manter o sigilo dos participantes e a confidencialidade das informações, as entrevistas foram codificadas com as letras "P", seguido da ordem em que os participantes foram entrevistados, por exemplo: "P11".

Foram realizadas entrevistas semiestruturadas, guiadas pela seguinte questão de pesquisa: "quais significados você atribuiu à gestão do grupo de pesquisa?". O diálogo do segundo grupo amostral foi instigado pela questão norteadora: "como você percebe a gestão do grupo de pesquisa?". As entrevistas foram áudio gravadas por meio de aplicativo de gravação de smartphone, armazenadas em um dispositivo portátil de armazenamento (pen drive) e transcritas na íntegra, utilizando o Microsoft ${ }^{\circledR}$ Office Word 2013. As entrevistas tiveram duração média de 38 minutos.

Após transcritas, as entrevistas foram encaminhadas via e-mail para validação, dois participantes solicitaram revisões relacionadas a redação do texto, não houve modificações do conteúdo das entrevistas. Nesse momento, também foi possível aprofundar dados, foi solicitado a um dos participantes para exemplificar detalhadamente o que ele queria dizer em determinada fala, visando a uma melhor compreensão do fenômeno em estudo. As entrevistas foram conduzidas pela pesquisadora principal do estudo, com experiência prévia com pesquisa qualitativa e coleta de dados na modalidade de entrevista. Acrescenta-se que no momento da coleta de dados, a pesquisadora era discente de Mestrado do Programa de Pós-Graduação em Enfermagem, em que o estudo foi realizado, mantendo contato acadêmico com os participantes da pesquisa.

A coleta de dados foi finalizada com a saturação teórica dos dados, ou seja, quando não foram evidenciados dados novos e as categorias estavam bem desenvolvidas quanto a propriedades, dimensões e validade. ${ }^{6}$

A análise dos dados seguiu os preceitos da perspectiva straussiana da Teoria Fundamentada nos Dados, por meio de codificação aberta, axial e integração. A codificação aberta é o momento de conceituação, em que os dados são agrupados de acordo com a sua similaridade, identificando as suas características e as propriedades inerentes a cada um dos conceitos. Em seguida, na codificação axial, obtêm-se as subcategorias, as quais são formadas a partir dos fenômenos iniciais, com vistas a explicação desses fenômenos. Por fim, a última categoria, é a integração, nesta se unificam os conceitos das categorias e subcategorias, a fim de construir a teoria. ${ }^{6}$ Os dados foram analisados e organizados com o apoio do software NVIVO ${ }^{\circledR} 10$.

Ao final, para organização dos dados, adotaram-se os três componentes do modelo paradigmático: condição, ações-interações e consequências. $O$ componente, condição, representa a causa expressada pelos participantes para a ocorrência de determinada situação, bem como suas explicações; as ações-interações são os movimentos, as conexões entre as pessoas com o intuito de buscar uma solução ou mudança para determinada situação; a partir da condição, das ações-interações surgem as consequências como resultado de tudo que foi gerado. Esses movimentos associados ao componente de análise evidencia o fenômeno. ${ }^{6}$

A partir da análise dos constructos, a direção analítica do estudo é conduzida pela construção de memorandos, explicitando o aprofundamento da hipótese, bem como a densidade teórica da pesquisa. O foco deste estudo é a subcategoria "Utilizando tecnologias de informação e comunicação", que se destacou pela relevância teórico-conceitual no componente "ações-interações", conforme o paradigma da codificação adotado. ${ }^{6}$

Esta pesquisa seguiu as recomendações descritas na Resolução $n^{\circ}$ 466, de 12 de dezembro de 2012, do Conselho Nacional de Saúde (CNS) e foi aprovado pelo Comitê de Ética em Pesquisa com Seres Humanos, com número de parecer 2.595.322 e Certificado de Apresentação para Apreciação Ética (CAAE) 81636317.0.0000.0121.

\section{RESULTADOS}

O estudo resultou na participação de 21 pesquisadores, 20 são do sexo feminino e um do sexo masculino, com idade entre 20 e 67 anos. O tempo de participação em grupos de pesquisa variou de 06 meses a 40 anos. Entre os líderes, vice-líderes e ex-líderes o tempo de atuação docente variou entre 12 e 53 anos, o tempo de atuação na pós-graduação entre 02 e 40 anos e o tempo de atuação como líder ou vice-líder foi de 01 a 30 anos. Para o pós-doutorando, doutorandos, mestrandos e graduandos a experiência com pesquisa variou de 03 meses a 11 anos.

As Tecnologias de Informação e Comunicação utilizadas pelos pesquisadores em grupos de pesquisa foi para facilitar os processos necessários de gestão, essenciais para o desenvolvimento das pesquisas, com três principais objetivos, comunicação e armazenamento, estratégias de visibilidade e estratégia operacional. A Figura 1 demonstra essas categorias e as estratégias inseridas em cada uma delas. 


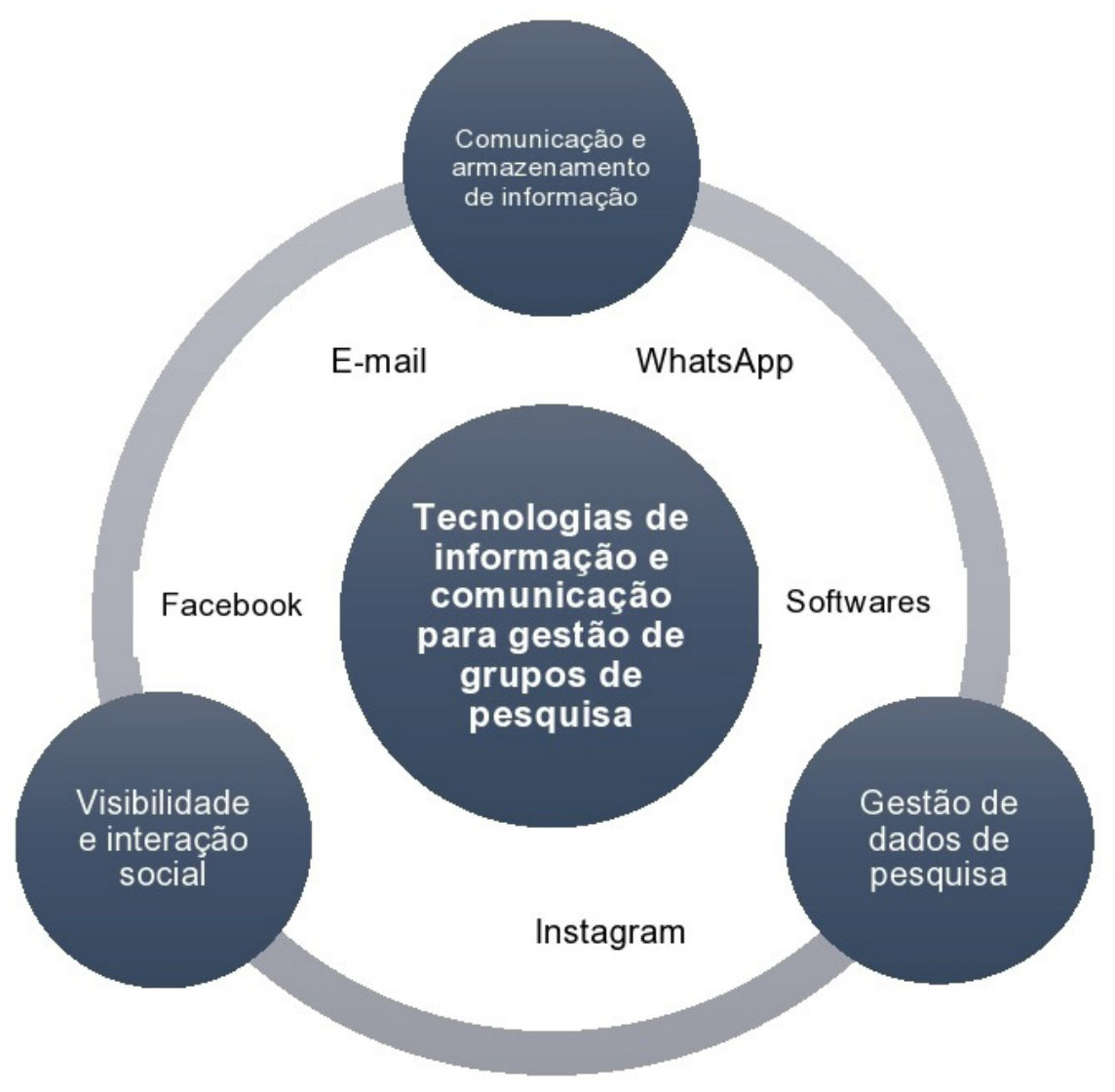

Figura 1. Tecnologias de Informação e Comunicação para a Gestão de Grupos de Pesquisa de Enfermagem. Elaborado pelos autores. Florianópolis, 2020.

\section{Comunicação e armazenamento de informações}

Os participantes evidenciaram que a comunicação se constitui como uma ferramenta crucial para auxiliar na gestão das atividades dentro dos grupos de pesquisa.

[...] Muitas vezes, falamos, genericamente, mas os processos de comunicação são realmente importantes e relevantes. Então, não é assim tão fácil se comunicar. Não é disso que eu estou falando, estou falando se pudéssemos pensar um processo efetivo de comunicação, acho que isso é um processo extremamente relevante para um grupo funcionar. Aítemos alguns mecanismos para se comunicar [...] (P13).

[...] Eu penso que a comunicação entre os membros dos grupos de pesquisa é realmente muito importante [...] (P16).

Para uma comunicação efetiva dentro dos grupos de pesquisa, os membros laçam mão do Whatsapp ${ }^{\circledR}$ e o e-mail como estratégias específicas de comunicação e armazenamento de informações. $O$ Whatsapp ${ }^{\circledR}$ é uma ferramenta utilizada para o compartilhamento de informações, como por exemplo: participação em congressos; compartilhamento de materiais; de notícias; de editais; de prazos. Além disso, foi utilizado também como um espaço para formação, para discussões e para a tomada de decisões compartilhadas. [...]o espaço do Whatsapp ${ }^{\circledR}$ do grupo tem bastante coisas e é um grupo que a gente compartilha muita informação e formação [...] (P13).

[...] temos um grupo de Whatsapp ${ }^{\circledR}$ também, éinteressante isso, não tem mensagens desnecessárias, mas sabemos quando alguém apresentou trabalho em eventos, por exemplo. Acho que depende da cooperação e participação de todo o grupo para acertar e afinar essa comunicação [...] (P16).

[...] tem a questão do grupo de Whatsapp ${ }^{\circledR}$ que vamos conversando sobre a data das reuniões do grupo e se muitas pessoas não podem em determinado dia mudamos para outro [...] (P18).

Em relação ao e-mail, também foi destacado como uma estratégia de comunicação e armazenamento. Nos grupos de pesquisa, foi utilizado para funções semelhantes as do Whatsapp ${ }^{\circledR}$, como facilitar o encaminhamento de avisos; de materiais e propiciar discussões. O e-mail institucional do grupo de pesquisa, também serviu como um espaço para armazenamento de informações.

[...] temos o espaço do e-mail. A professora líder manda tudo por e-mail [...] (P16). 
[...] utilizamos o e-mail coletivo também do grupo, por exemplo quando fizemos um evento, enviamos um formulário de inscrição e sempre enviamos em anexo para o e-mail do grupo, então sempre conseguimos resgatar as coisas de eventos passados. Quando vamos fazer um novo evento, por exemplo, pegamos o que já foi utilizado e fizemos uma crítica se aquilo ainda é válido, se foi bem aplicado, se foi efetivo com o que queríamos, e aplicamos de novo, ou não, com alteração. Então, a gente sempre utiliza o e-mail do grupo como um lugar para mandar coisas e guardar para que todo mundo consiga ter acesso [...] (P19).

\section{Divulgação e visibilidade}

As estratégias de visibilidade citadas destacaram o uso do site e das mídias sociais, como Facebook ${ }^{\circledR}$ e Instagram ${ }^{\circledR}$. Em relação ao site, essa já é uma prática existente e sistematizada por alguns grupos de pesquisa. Entretanto, outros grupos ainda enfrentam dificuldades para a criação da página eletrônica, principalmente devido a restrições orçamentárias.

Tanto para os grupos que já possuem um site, quanto para os grupos que estão implementando, a atualização constante dessas páginas foi citada como um desafio, pois depende do envolvimento de docentes e discentes. Um dos fatores que dificulta essa solução é a rotatividade dos discentes de graduação e pós-graduação, cujo tempo de permanência no grupo de pesquisa corresponde, muitas vezes, somente ao período de formação.

Os grupos que conseguem manter seus sites atualizados centralizam essa tarefa em membros específicos, os quais são responsáveis por atividades, como: calendário de reuniões, participação em eventos, próximos eventos, publicações, entre outros. É consenso para os participantes dos grupos de pesquisa que a manutenção de um site atualizado aumenta a visibilidade desses Grupos e a sua repercussão para além dos muros da universidade.

[...] nós também temos o site do grupo de pesquisa, que é um site institucional, é da universidade, é nomedogrupodepesquisa.universidade.br. É difícil um grupo de pesquisa, um laboratório ter o seu site próprio não é? O que nós precisamos fazer agora é essa atualização, só que o nosso tempo está curto. Então, a gente vai ficar dependendo dos alunos para atualizar, que já tem as suas atividades. É nosso projeto estar sempre pensando alguém que possa fazer essa atualização, que também depende de todos os professores estar colaborando [...] (P1).

[...] não temos uma página no site da universidade ainda. Não tivemos recurso financeiro para montar essa página. Tem que ter alguém que saiba colocar, o mais difícil é montar a página porque a manutenção somos nós mesmos que fazemos. Mas, eu imagino que a partir desse próximo semestre a gente vai dar isso como uma prioridade. Então, agora a gente está vendo que isso é importante, nós estamos ficando meio que defasados porque a gente não tem um espaço de divulgação, a página é um espaço de divulgação. E aí isso acaba comprometendo a divulgação do próprio grupo, não é só com eventos que a gente propaga o grupo, mas também é em uma página, são as reuniões, e aí é isso que a gente tem procurado fazer [...] (P9).

As mídias sociais relatadas foram o Facebook ${ }^{\circledR}$ e o Instagram ${ }^{\circledR}$, com o intuito de aumentar a visibilidade do trabalho e das pesquisas dentro dos Grupos. O uso de mídias sociais surgiu a partir de uma necessidade identificada por membros dos grupos de pesquisa em enfermagem. Como nesses grupos de pesquisa, a gestão ocorre de forma compartilhada entre os membros, a ideia foi acolhida e viabilizada por todos os seus participantes.

Essa estratégia foi pensada principalmente por aliar mídias sociais que fazem parte da vida das pessoas. É muito comum o acesso a essas plataformas diariamente. Os participantes também relataram que facilita a conexão entre as pessoas afastadas geograficamente, como por exemplo, ex-participantes ou participantes ativos que não podem estar presentes fisicamente nas reuniões, mas acompanham o que vem sendo trabalhada, as pesquisas realizadas, as defesas. Além disso, para quem ainda não conhece o grupo de pesquisa, as mídias sociais possibilitam essa aproximação e o conhecimento de como é o grupo de pesquisa.

[...] revitalizamos a página do Facebook ${ }^{\circledR}$ e criamos a conta do Instagram ${ }^{\circledast}$. Muitos criticam a utilização das redes sociais em um sentido mais de explicitação da vida privada. Quando penso no papel da universidade e da democratização do conhecimento, penso que devemos compartilhar mais o que acontece no ambiente acadêmico. Quantos amigos nossos não sabem das teses e dissertações que trabalhamos, ou ainda acham que estudar é uma tarefa fácil, não sendo entendido como trabalho. Então, penso que utilizar as redes sociais é uma forma de dar visibilidade para o nosso fazer, dialogando com ferramentas mais contemporâneas. $E$ isso conecta pessoas. Temos uma rede no Brasil e fora e isso permite que estas pessoas saibam o que tá acontecendo no grupo de pesquisa. Aí a ideia veio dessa gestão coletiva que vem acontecendo e de como podemos abrir a porta do grupo pra todas e todos [...] (P13).

[...] tem sido bem bacana reconectar as pessoas. Além disso, o Instagram utiliza o recurso fotográfico, que por si só já significa. Então, isso afeta as pessoas [...] (P13).

\section{Gestão de dados de pesquisa}

Para a gestão de dados de pesquisa, foi levantado o uso de softwares que servem como apoio para os pesquisadores na organização e análise de dados proveniente das suas pesquisas. 
Os grupos de pesquisa dispõem de softwares para organização e análise de dados qualitativos e dados quantitativos. Os softwares de análise de dados qualitativos mais citados foram o NVIVO ${ }^{\circledR}$, MAXQDA ${ }^{\circledR}$, Atlas.ti ${ }^{\circledR}$ e para a análise de dados quantitativos o IBM SPSS ${ }^{\circledR}$ Statistics.

[...] eu tive oportunidade, de conhecer, por exemplo, o software NVIVO que a gente tem ali no grupo de pesquisa $[\ldots](P 11)$.

\section{DISCUSSÃO}

A academia vem gerando novas tendências e inovações juntamente ao desenvolvimento científico e tecnológico. Os grupos de pesquisa contribuem para a produção científica do país por meio das publicações e do intercâmbio de informações e experiências entre os pesquisadores. ${ }^{7} \mathrm{Na}$ formação de pesquisadores em cursos de pós-graduação Stricto sensu docente, mais especificamente em estágio de docência, ressalta-se a importância da compreensão das tecnologias disponíveis e a apropriação de recursos tecnológicos como aliados para o processo de ensino e aprendizagem. ${ }^{8}$

A partir dos constructos teóricos apresentados nos resultados do estudo foi possível relacionar os achados com a literatura científica, a qual corrobora com os dados do estudo ao descrever os aspectos positivos da aplicabilidade das Tecnologias da Informação e Comunicação nos grupos de pesquisa, no que tange as Tecnologias da Informação e Comunicação produtoras de conhecimento e as de suporte ao desenvolvimento do conhecimento. As primeiras estão relacionadas às tecnologias voltadas à educação, ao cuidado e ao desenvolvimento de software. A segunda, ligada a organização das produções do grupo: armazenamento, disseminação e divulgação. A sinergia entre tecnologia e ciência da vida leva ao avanço de uma nova biologia. ${ }^{4}$

O Whatsapp ${ }^{\circledR}$ citado pelos participantes do estudo, também foi descrito de maneira semelhante na literatura como uma ferramenta efetiva de auxílio ao gerenciamento de informações de grupos. Um laboratório de análises clínicas incluiu os trabalhadores nos grupos de Whatsapp ${ }^{\circledR}$ correspondente ao setor em que trabalhavam. As informações relacionadas com os serviços de laboratório foram compartilhadas nos grupos. O uso comedido do aplicativo apresentou melhora significativa na comunicação, facilitada pelo compartilhamento de fotos, alertas críticos, informações sobre acidentes, escalas, atividades acadêmicas e orientações. Em decorrência disso houve um aumento na carga de informações que atrapalhou minimamente a rotina, todavia os benefícios foram superiores a essas situações. ${ }^{9}$

As mídias sociais têm sido cada vez mais utilizadas como aliadas dos pesquisadores, por oportunizarem o acesso a banco de dados. O seu uso está atrelado ao aumento da visibilidade da pesquisa e recentemente está sendo utilizada como um método efetivo e econômico de recrutamento de participantes para novas pesquisas. Além disso, essa forma de recrutamento favorece a diversidade geográfica, social e econômica dos participantes, bem como viabiliza a participação de populações de difícil acesso e a construção de uma amostra robusta. Esse modelo de recrutamento de participantes para a pesquisa demonstra que ao longo do tempo os grupos de pesquisa desenvolvem novos métodos, modelos e teorias com o apoio das telecomunicações, com o intuito de potencializar a infraestrutura já disponível, visando novas aplicações e serviços. ${ }^{3-10}$

Os softwares de apoio à análise de dados podem ser descritos como uma estratégia de gestão de dados de pesquisa, pois exercem a função de organizar de forma sistemática e ágil o armazenamento, o gerenciamento e a recuperação dos dados. Embora os softwares sejam importantes para esses processos, a capacidade analítica do pesquisador é indispensável para conduzir o pensamento analítico e a condução da pesquisa científica. O software serve como um importante apoio para a organização e análise dos dados e para a finalização dos resultados de pesquisa. ${ }^{11}$ Os pesquisadores em ciências da saúde, discentes de pós-graduação e professores, consideram importante abordar o uso de softwares para apoio a análise de dados em um treinamento em Tecnologia da Informação e Comunicação. ${ }^{3}$

Nesse contexto de inserção de Tecnologias da Informação e Comunicação em Grupos de pesquisa, o foco dos estudos passa a considerar a necessidade de um planejamento e o controle de cyber sistemas, como logística humanitária, redes inteligentes, cadeias multimodais de abastecimento e a regulação de sistemas sociais e biológicos. Além disso, são incluídos escolha social computacional, computação pervasiva, diagnóstico, previsão e simulação como tópicos fundamentais para a pesquisa. ${ }^{4}$

Estudo realizado com 1.065 discentes de pós-graduação, identificou que a maioria dos estudantes (69\%) utilizam a Tecnologia da Informação e Comunicação nas pesquisas que estavam desenvolvendo e (31\%) não utilizavam nenhum tipo de Tecnologia de Informação e Comunicação. Entretanto, mais de $65 \%$ reconheceram a importância de abordar a temática de uso de Tecnologia da Informação e Comunicação, bem como, a realização de um treinamento em uso de Tecnologia da Informação e Comunicação na pesquisa. Os discentes também citaram a relevância do conhecimento sobre a Tecnologia da Informação e Comunicação como essenciais para suas necessidades educacionais, para auxiliar os pesquisadores na sua eficiência e eficácia; para desenvolver protocolos de pesquisa; na utilização de registros eletrônicos em saúde para pesquisa; conhecimento sobre o funcionamento de bancos de dados (estrutura, atributos, armazenamento, recuperação e relacionamento dos dados); como utilizar as Tecnologias da Informação e Comunicação em cada fase da pesquisa. ${ }^{3}$

Na literatura, é possível encontrar instrumentos para avaliar a atitude de discentes das áreas de ciências da saúde frente ao uso de tecnologias, que facilitam a tomada de decisão a partir do acesso à informação e da gestão da informação. Como por exemplo, a Escala de atitudes em tecnologias digitais da informação e comunicação à saúde. A escala favorece a gestão 
da informação, bem como a administração das tecnologias da informação na educação, fornecendo elementos a pesquisadores e gestores para aliar-se a essas tecnologias na busca por qualificação na prática assistencial em saúde. ${ }^{12}$

Os grupos de pesquisa em enfermagem podem ainda incorporar novas ferramentas com o apoio da Tecnologias da Informação e Comunicação, já empregada em outros países, para a condução de pesquisas colaborativas que resultam em maior robustez e melhor acurácia. No que tange ao potencial de colaboração em pesquisa em saúde a nível global, o uso das Tecnologias da Informação e Comunicação permitiu diversas formas de interação entre pesquisadores, como por exemplo: publicações em conjunto; compartilhamento de banco de dados em pesquisas; mentorias; apoio a grupos de pesquisa; colaboração e financiamento de projetos pilotos. ${ }^{13}$

Além disso, o uso das Tecnologias da Informação e Comunicação em grupos de pesquisa potencializa o apoio ao fortalecimento da pesquisa em países emergentes, ao conectar cientistas da saúde dos dois extremos e facilitar o acesso a instrumentalização em pesquisa em saúde por meio de ferramentas de ensino a distância. ${ }^{13} \mathrm{Na}$ Noruega, o desenvolvimento de pesquisa em redes internacionais de colaboração apresentou repercussões na melhoria da qualidade das publicações e no aumento da produtividade dos pesquisadores. ${ }^{14}$

No Canadá, são descritas parcerias entre pesquisadores e a comunidade interessada, como pacientes, organizações comunitárias, profissionais e formuladores de políticas, o que gera um impacto para todos os participantes envolvidos, bem como para o desenvolvimento de pesquisas bem-sucedidas. ${ }^{15}$

As Tecnologias da Informação e Comunicação são importantes ferramentas para a melhoria da educação em saúde global. A aplicação desses dispositivos ganha, cada vez mais, um espaço fundamental no ambiente de ensino e aprendizado das áreas da saúde, em um cenário de profundas transformações e digitalização da saúde, com a transição para atendimentos, diagnóstico e tratamento de forma remota. As Tecnologias da Informação e Comunicação favorecem o intercâmbio de informações sobre a saúde, bem como treinamentos em saúde a nível global; possibilita a colaboração entre pesquisadores; fornece instrumentos para a tomada de decisão em políticas públicas de saúde. ${ }^{3}$

\section{CONCLUSÃO}

As Tecnologia da Informação e Comunicação utilizadas por Grupo de Pesquisa da Enfermagem auxiliam os seus processos de gestão de ciência, tecnologia e inovação em saúde, bem como constroem um elo entre tecnologia e construção do conhecimento científico, tecnológico e de inovação. A partir dos construtos resultantes da centralidade dos achados, o estudo aponta como inovação a utilização das Tecnologias da Informação e Comunicação com três funções principais dentro dos grupos de pesquisa: comunicação e armazenamento de informação, divulgação e visibilidade e gestão e armazenamento de dados.

Embora os grupos de pesquisa façam uso de uma gama de Tecnologia da Informação e Comunicação, podem ampliar o seu arsenal, haja visto a quantidade de recursos disponíveis nesse sentido. Como por exemplo, utilizar-se das mídias sociais para o recrutamento e seleção de amostra para pesquisas futuras $\mathrm{e}$ o desenvolvimento de redes colaborativas de pesquisa.

O estudo apresentou limitações relacionadas a escassez de literatura sobre o uso das Tecnologias da Informação e Comunicação específicas em grupos de pesquisa, por mais que as Tecnologias da Informação e Comunicação sejam rotineiras no dia a dia de pesquisadores, foi um fator limitante. Assim, evidencia-se a necessidade de novas investigações para aprofundamento da temática investigada e divulgação do potencial das Tecnologias da Informação e Comunicação para a gestão de grupos de pesquisa e produção do conhecimento científico.

\section{FINANCIAMENTO}

O presente trabalho contou com apoio da Coordenação de Aperfeiçoamento de Pessoal de Nível Superior - Brasil (CAPES) - Código de Financiamento 001.

Bolsa de doutorado concedida a Greici Capellari Fabrizzio pelo Conselho Nacional de Desenvolvimento Científico e Tecnológico (CNPQ).

Bolsa de Produtividade em Pesquisa - Nível 1A, concedida pelo Conselho Nacional de Desenvolvimento científico e Tecnológico (CNPQ) a Alacoque Lorenzini Erdmann.

\section{CONTRIBUIÇÕES DOS AUTORES}

Desenho do estudo. Greici Capellari Fabrizzio. Juliana Martins Ferreira. Daniele Cristina Perin. Patricia Klock. Alacoque Lorenzini Erdmann. José Luís Guedes dos Santos

Coleta ou produção dos dados. Greici Capellari Fabrizzio. Juliana Martins Ferreira. Daniele Cristina Perin. Patricia Klock. Alacoque Lorenzini Erdmann. José Luís Guedes dos Santos

Análise de dados. Greici Capellari Fabrizzio. Juliana Martins Ferreira. Daniele Cristina Perin. Patricia Klock. Alacoque Lorenzini Erdmann. José Luís Guedes dos Santos

Interpretação dos resultados. Greici Capellari Fabrizzio. Juliana Martins Ferreira. Daniele Cristina Perin. Patricia Klock. Alacoque Lorenzini Erdmann. José Luís Guedes dos Santos

Redação e revisão crítica do manuscrito. Greici Capellari Fabrizzio. Juliana Martins Ferreira. Daniele Cristina Perin. Patricia Klock. Alacoque Lorenzini ErdmanN. José Luís Guedes dos Santos

Aprovação da versão final do artigo. Greici Capellari Fabrizzio. Juliana Martins Ferreira. Daniele Cristina Perin. Patricia Klock. Alacoque Lorenzini Erdmann. José Luís Guedes dos Santos

Responsabilidade por todos os aspectos do conteúdo e a integridade do artigo publicado. . Greici Capellari Fabrizzio. Juliana Martins Ferreira. Daniele Cristina Perin. Patricia Klock. Alacoque Lorenzini Erdmann. José Luís Guedes dos Santos

\section{EDITOR ASSOCIADO}

Candida Caniçali Primo 


\section{REFERÊNCIAS}

1. Moreno Cely GA, Gutiérrez Rodrígues RE. Prospective study of thecnology in Higher Education in Colombia by 2050. Rev. Univ. Empresa.2020;22(38):1. http://dx.doi.org/10.12804/revistas.urosario. edu.co/empresa7a.7583.

2. Conselho Nacional de Desenvolvimento Científico e Tecnológico, Diretório dos Grupos de Pesquisa no Brasil. Súmula estatística [Internet]. Brasília (DF); 2018. [citado 2020 mar 15]. Disponível em: http://lattes. cnpq.br/web/dgp/por-grande-area1

3. Huang K, Abdullah AS, Ma Z, Urmi DS, He H, Quintiliani L et al. Attitudes of Chinese health Sciences postgraduate students' to the use of information and communication technology in global health research. BMC Med Educ. 2019;19(1):367. http://dx.doi.org/10.1186/ s12909-019-1785-6.

4. Erdmann AL, Santos JLG, Klock P, Soder RM, Dal Sasso GTM, Erdmann $\mathrm{RH}$. Policies, management and innovation research groups for nursing excellence. Aquichan. 2013;13(1):92-103. http://dx.doi.org/10.5294/ aqui.2013.13.1.8.

5. Galindo No NM, Áfio ACE, Leite SS, Silva MG, Pagliuca LMF, Caetano JA. Technologies for health education for the deaf: integrative review. Texto Contexto Enferm. 2019;28:e20180221. http://dx.doi.org/10.1590/1980265x-tce-2018-0221.

6. Corbin J, Strauss A. Basics of qualitative research: techniques and procedures for developing Grounded Theory. California: SAGE; 2015.

7. Domingues AN, Jesus ITM, Zem-Mascarenhas SH. Informática na Educação em Saúde e Enfermagem: análise dos grupos de pesquisa. J Health Inform [Internet]. 2017 Mar; [citado 2020 mar 15];9(1):19-24. Disponível em: http://www.jhi-sbis.saude.ws/ojs-jhi/index.php/jhi-sbis/ article/view/460
8. Alves LR, Giacomini MA, Teixeira VM, Henriques SH, Chaves LP. Refletions on graduate professor training. Esc Anna Nery. 2019;23(3):e20180366. http://dx.doi.org/10.1590/2177-9465-ean-2018-0366.

9. Dorwal P, Sachdev R, Gautam D, Jain D, Sharma P, Tiwari AK et al Role of WhatsApp Messenger in the laboratory management system: a boon to communication. J Med Syst. 2016;40(1):14. http://dx.doi. org/10.1007/s10916-015-0384-2.

10. Burgess JD, Kimble RM, Watt K, Cameron CM. The adoption of social media to recruit participants for the cool runnings randomized controlled trial in Australia. JMIR Res Protoc. 2017 out;6(10):e200. http://dx.doi. org/10.2196/resprot.8189.

11. Nunes JV, Woloszyn M, Gonçalves BS, Pinto MDS. A pesquisa qualitativa apoiada por softwares de análise de dados: uma investigação a partir de exemplos. Rev Front. 2017 Aug;19(2):233-244. http://dx.doi.org/10.4013/ fem.2017.192.08.

12. Oliveira MR, Correia VG, Dantas EO, Moreira TM, Torres RA. Validation of the atitude scale for information and communications Technologies. Acta Paul Enferm. 2019;32(1):79-86. http://dx.doi.org/10.1590/19820194201900011.

13. Wang J, Abdullah AS, Ma Z, Fu H, Huang K, Yu H et al. Bulding capacity for information and communication technology use in global health research and training in China: a qualitative study among Chinese health sciences faculty members. Health Res Policy Syst. 2017 Jun;15(1):59 http://dx.doi.org/10.1186/s12961-017-0222-8.

14. Kyvik S, Reymert I. Research collaboration in groups and networks: differences across academic fields. Scientometrics. 2017 set;113(2):95167. http://dx.doi.org/10.1007/s11192-017-2497-5.

15. Hoekstra F, Martin Ginis KA, Allan V, Kothari A, Gainforth HL. Evaluating the impact of a network of research partnerships: a longitudinal multiple case study protocol. Health Res Policy Syst. 2018;16(1):107. http:// dx.doi.org/10.1186/s12961-018-0377-y. 